\title{
Regional cortical thinning of the orbitofrontal cortex in medication-naïve female patients with major depressive disorder is not associated with MAOA-uVNTR polymorphism
}

\author{
Eunsoo Won ${ }^{1}$, Sunyoung Choi ${ }^{2}$, June Kang ${ }^{3}$, Min-Soo Lee ${ }^{4}$ and Byung-Joo Ham*
}

\begin{abstract}
Background: Orbitofrontal cortex alterations have been suggested to underlie the impaired mood regulation in depression. MAOA-uVNTR (monoamine oxidase A-upstream variable number of tandem repeats) polymorphism has been reported to be associated with major depressive disorder by various studies. The influence of MAOA-uVNTR genotype on function and structure of the orbitofrontal cortex has previously been reported. In this study, we investigated the difference in orbitofrontal cortex thickness between medication-naïve female patients with major depressive disorder and healthy controls, and the influence of MAOA-uVNTR genotype on orbitofrontal cortex thickness in depression.
\end{abstract}

Methods: Thirty-one patients with major depressive disorder and 43 healthy controls were included. All participants were subjected to T1-weighted structural magnetic resonance imaging and genotyped for MAOA-uVNTR polymorphism. An automated procedure of FreeSurfer was used to analyze difference in orbitofrontal cortex thickness.

Results: Patients showed a significantly thinner left orbitofrontal cortex $\left(F_{(1,71)}=7.941, p=0.006\right)$ and right orbitofrontal cortex $\left(F_{(1,71)}=17.447, p<0.001\right)$. For the orbitofrontal cortex sub-region analysis, patients showed a significantly thinner left medial orbitofrontal cortex $\left(F_{(1,71)}=8.117, p=0.006\right)$, right medial orbitofrontal cortex $\left(F_{(1,71)}=21.795, p<0.001\right)$ and right lateral orbitofrontal cortex $\left(F_{(1,71)}=9.932, p=0.002\right)$ compared to healthy controls. No significant interaction of diagnosis and MAOA-uVNTR genotype on orbitofrontal cortex thickness was revealed.

Conclusions: Our results suggest that structural alterations of the orbitofrontal cortex may be associated with the pathophysiology of major depressive disorder. Future studies with larger sample sizes are needed to detect a possible association between MAOA-uVNTR genotype and orbitofrontal cortex thickness in depression.

Keywords: Major depressive disorder, Orbitofrontal cortex thickness, Monoamine oxidase A-upstream variable number of tandem repeats

\section{Background}

Neuroimaging studies on major depressive disorder (MDD) have consistently identified neuroanatomical alterations in brain regions that participate in affect

\footnotetext{
*Correspondence: hambj@korea.ac.kr

${ }^{1}$ Department of Psychiatry, Korea University Anam Hospital, Korea University College of Medicine, 73, Inchon-ro, Seongbuk-gu, Seoul 136-705, Republic of Korea

Full list of author information is available at the end of the article
}

regulation, such as the prefrontal cortex and limbic system [1]. The prefrontal cortex is known to be a heterogeneous structure both anatomically and functionally and it has been suggested that specific subdivisions of the prefrontal cortex, for instance, the orbitofrontal cortex (OFC), may be differently involved in the pathophysiology of MDD [2]. The OFC is considered to play an important role in mood regulation, and damage to the OFC has been characterized by deficits in emotion and social 
regulation along with mood lability [3]. Therefore, numerous structural neuroimaging studies have been conducted on the OFC in MDD patients, with studies using whole-brain approaches with voxel-based morphometry reporting gray matter volume reduction of the OFC in MDD patients [4]. In addition, as the OFC itself has been reported to be regionally specialized [5], studies that have separately evaluated both medial and lateral OFC volumes in MDD have reported reduced gray matter volumes in both areas [2]. Studies measuring cortical thickness have reported an association between MDD and cortical thinning of the lateral OFC [6] and medial OFC [7].

Despite these many reports, it is not yet clear what factors may contribute to the alteration of OFC structure in MDD. Genetic polymorphisms that increase the risk for depression are considered to be one of the contributors, as these genetic variants are presumed to act on brain function and architecture [8]. Polymorphisms of the monoamine oxidase A $(M A O A)$ gene have been associated with an increased risk of depression [9]. An upstream variable number of tandem repeats (uVNTR) polymorphism in the promoter region of the $M A O A$ gene consists of a 30-bp repeat sequence present in 2, 3, $3.5,4$, or 5 repeats $(R)$ [10]. As the $3.5 R$ or $4 R$ alleles were reported to be transcribed 2-10 times more efficiently than $3 \mathrm{R}$ or $5 \mathrm{R}$ alleles, previous studies have defined $M A O A-\mathrm{uVNTR}$ polymorphism to produce genotypes with low activity and high activity [11]. Although highactivity $M A O A$-uVNTR variants $(M A O A-\mathrm{H}$, genotypes associated with $3.5 \mathrm{R}$ or $4 \mathrm{R}$ alleles) have been associated with MDD [12], some studies have failed to find such an association [13], and various studies have reported an association between low-activity $M A O A$-uVNTR variants $(M A O A-\mathrm{L}$, genotypes associated with $3 \mathrm{R}$ or $5 \mathrm{R}$ alleles) and MDD [9].

Monoamine oxidase A-upstream variable number of tandem repeats polymorphism has also been associated with structural alterations of brain regions involved in mood regulation [14]. How MAOA modulates brain structure has previously been explained by the role of MAOA enzyme in regulating the metabolism of monoamine neurotransmitters [15]. Therefore, numerous imaging genetics studies have been conducted on $M A O A$-uVNTR polymorphism, with a majority reporting $M A O A$-uVNTR genotype to be relevant to OFC structure in healthy controls (HCs). Decreased OFC activity during perceptual matching of angry and fearful faces in healthy individuals with $M A O A-\mathrm{L}$, along with increased OFC volume in male $M A O A-\mathrm{L}$ subjects, has previously been reported [16]. Reduction of OFC volume in $M A O A-\mathrm{H}$ HCs was also reported [17], whereas $M A O A-\mathrm{H}$ subjects were shown to have the highest OFC thickness in another study [18].
Cortical thickness refers to the grey matter of the cortex, and shrinkage of neurons, reduction of synaptic spines and lower number of synapses may account for the reduction in grey matter, which may lead to the decrease in function of the thinned area [19]. Whereas measurement of volume may give insufficient information about the dimensions of structure, measurement of thickness enables a continuous measurement across the cortical surface [20], and might be more sensitive to detect structural abnormalities [21]. It has also been suggested that cortical thickness measurements should be preferred over gray matter volume measurements for imaging genetics studies [22]. FreeSurfer has been reported as a highly reliable method for automated cortical thickness measurement [23].

Therefore, we hypothesized MDD patients to exhibit a reduction in OFC thickness compared to HCs. We also hypothesized $M A O A$-uVNTR polymorphism to have influence on the alteration of OFC thickness in MDD, which will be observed as a significant interaction of diagnosis (MDD patients, $\mathrm{HCs}$ ) and $M A O A-\mathrm{uVNTR}$ genotype (MAOA-H, $M A O A-\mathrm{L})$ on OFC thickness. The neurotrophic effects of antidepressants, which may reverse neuronal atrophy, have previously been reported [24]. Therefore, only medication-naïve MDD patients, who had never taken psychotropic medications before, were included in this study when comparing OFC thickness. In addition, MDD is known to be more prevalent among females, and as the $M A O A$ gene is located on the $\mathrm{X}$ chromosome and sex-specific effects of $M A O A$ have previously been reported [25], our patient and control groups only consisted of females.

\section{Methods}

Participants

We studied 31 medication-naïve female patients with MDD and 43 HCs. Patients were recruited from the outpatient psychiatric clinic of Korea University Anam Hospital located in Seoul, Republic of Korea. Diagnosis was determined by a board-certified psychiatrist, according to the Diagnostic and Statistical Manual for Mental Disorders-IV-Text Revision (DSM-IV-TR), using the Korean version of the Structured Clinical Interview for DSM-IV. Severity of depression was measured by the 17-item Hamilton Depression Rating Scale (HRDS) on the day of magnetic resonance imaging (MRI) acquisition. Patients with primary or comorbid psychiatric diagnoses other than MDD were excluded from the study. Patients suffering from serious or unstable medical illness and primary neurological illness were also excluded. Forty-three age, sex, and education level matched HCs were recruited by advertisements from the community. HCs were screened for major psychiatric histories, and 
none had a psychiatric disorder. The age of subjects in both groups ranged from 23 to 60 years. All subjects were right-handed as revealed by the Edinburgh Handedness Test and were self-identified Koreans with ethnic origin ascertained by confirming the ethnicity of three generations of the patients' families. The protocol was approved by the institutional review board of Korea University College of Medicine and signed informed consent was obtained from all participants according to the Declaration of Helsinki.

\section{MRI acquisition}

Three-dimensional structural MRI scans were acquired from a $3.0 \mathrm{~T}$ Siemens Trio whole-body imaging system (Siemens Medical Systems, Iselin, NJ, USA), using a T1-weighted magnetization-prepared rapid gradientecho (MP-RAGE $1900 \mathrm{~ms}$ repetition time, $2.6 \mathrm{~ms}$ echo time, $220 \mathrm{~mm}$ field of view, $256 \times 256$ matrix size, 176 coronal slices without gap, $1 \times 1 \times 1 \mathrm{~mm}^{3}$ voxels, $16^{\circ}$ flip angle, number of excitations $=1$ ). All scans were inspected for motion artifacts and a neuroradiologist confirmed the absence of gross pathological findings.

\section{MR scan processing and calculation of cortical thickness}

Cortical thickness analyses were performed on the three-dimensional model of cortical surface reconstructions computed from T1 images using the FreeSurfer 5.0 software package (Massachusetts General Hospital, Boston, U.S., http://surfer.nmr.mgh.harvard.edu). The details of technical aspects in these procedures have been described in previous publications [26-30]. Briefly, the implanted processing stream involved motion correction of volumetric T1-weighted images, removal of non-brain tissue using a hybrid watershed/surface deformation procedure, automated Talairach transformation of each subject's native brain, segmentation of the gray matterwhite matter volumetric structures, inflation of cortical surface to an average spherical surface to locate both the pial surface and the gray matter-white matter boundary, intensity normalization, and automated topology correction. Transition of gray/white matter and pial boundary was indicated by detecting the greatest shift in intensity through surface deformation. The cortex of each subject was then visually inspected. The entire cerebral cortex was parcelated into units based on gyral and sulcal structure. The computed cortical thickness was defined as the shortest distance between the pial surface and the gray matter-white matter boundary at each given point across the cortex. The cortical maps were generated by computing mean cortical thickness for each subject at each vertex, right and left hemispheres separately, and mapping these data to the surface of an average brain template enabling visualization of data across the entire cortical surface. Smoothing with a Gaussian kernel of $10 \mathrm{~mm}$ full width at half-maximum was performed on the cortical maps of each subject for the entire cortex analyses.

\section{Calculation of averaged cortical thickness within region of interest}

This study focused on the difference in cortical thickness within a region of interest (ROI), the OFC. As the OFC has been reported to be regionally specialized, cortical thickness of the medial and lateral sub-regions of the OFC was also separately calculated [5]. The ROI was defined with reference to the Desikan-Killiany atlas (Fig. 1) [30], and the FreeSurfer package automatically estimated individual cortical thickness of the ROI for the left and right hemisphere by mapping each ROI mask. Cortical thickness measurement of each vertex of the subjects' surface was mapped on a common spherical coordinate system using a spherical transformation.

\section{Genetic analyses and identification of subgroups}

Patients and HCs were genotyped for $M A O A$-uVNTR polymorphism. Venous blood was drawn from each subject using a protocol approved by the Ethics Committee of the Korea University Medical Center. DNA was isolated using standard techniques. Genotyping of the MAOA-uVNTR polymorphism was performed as described by Manor et al. [31]. According to genotype, patients and $\mathrm{HCs}$ were divided into two subgroups: $M A O A$-uVNTR high-activity allele carriers $(3 \mathrm{R} / 4 \mathrm{R}$, 4R/4R, $M A O A-\mathrm{H})$ versus homogenous $M A O A-\mathrm{uVNTR}$ low-activity allele carriers (2R/3R, 3R/3R, MAOA-L). Although heterozygous females with one or two lowactivity alleles have been grouped as $M A O A-\mathrm{L}$ in some studies, others have grouped such subjects as $M A O A-\mathrm{H}$ [9], which is in accordance with our subgrouping.

\section{Statistical analysis}

Differences in demographic and clinical characteristics between MDD patients and HCs were analyzed using one-way ANOVA for continuous variables (age, years of education, illness duration and HDRS scores). HardyWeinberg equilibrium (HWE) of allele frequencies of $M A O A$-uVNTR was analyzed using a Chi-square test. The difference in averaged cortical thickness $(\mathrm{mm})$ of the OFC, including the medial and lateral sub-regions, between MDD patients and HCs was analyzed using ANCOVA with age as a covariate. When comparing the OFC as a whole, the right and left OFC were separately tested, at $p<0.05 / 2=0.025$ for multiple comparisons with Bonferroni correction. When comparing the sub-regions of the OFC, 4 sub-regions (left medial OFC, left lateral OFC, right medial OFC, and right lateral OFC) were separately tested at $p<0.05 / 4=0.0125$ 


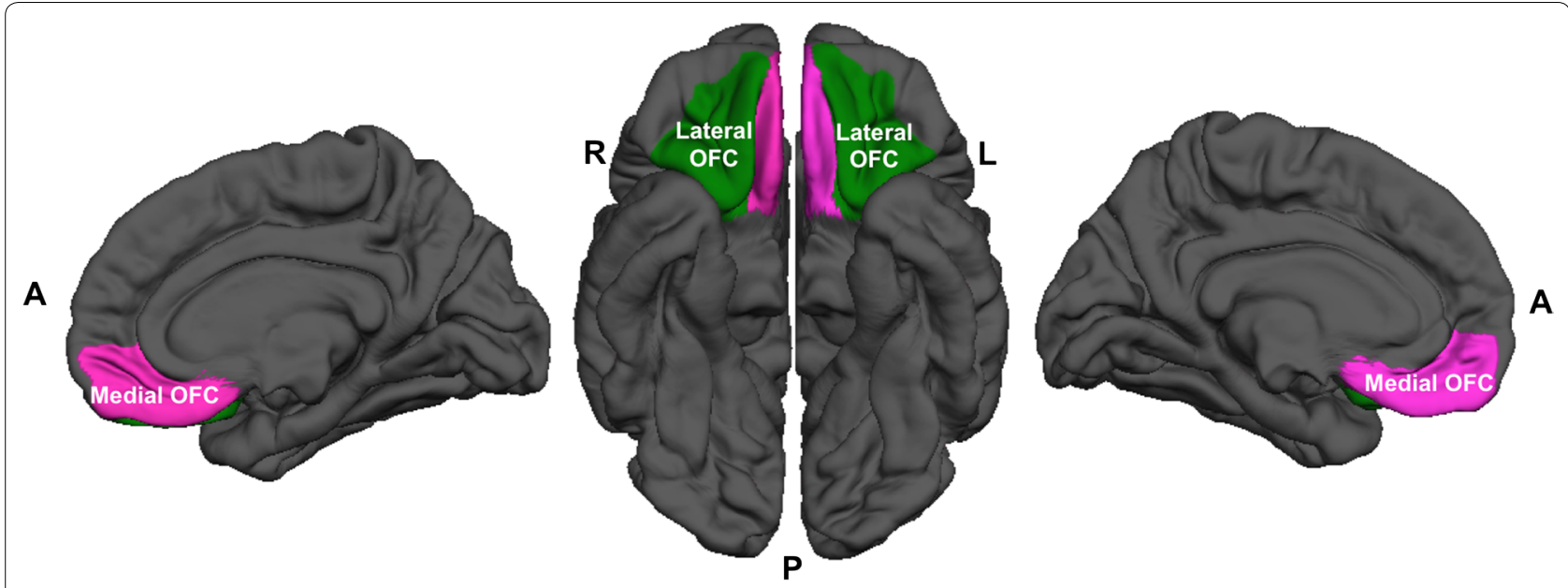

Fig. 1 The orbitofrontal cortex including the medial and lateral sub-regions, defined with reference to the Desikan-Killiany atlas

after Bonferroni correction. The interaction of diagnosis (MDD patients, $\mathrm{HCs}$ ) and $M A O A$-uVNTR genotype (MAOA-H, MAOA-L) on OFC thickness was examined using ANCOVA with age as a covariate.

\section{Results}

\section{Demographic and clinical characteristics}

Age, years of education, duration of illness, and HDRS scores of $31 \mathrm{MDD}$ patients and $43 \mathrm{HCs}$ are shown in Table 1. No significant difference in age and years of education was found between MDD patients and HCs. The allele frequencies of MAOA-uVNTR in MDD patients and HCs were in agreement with the HWE as shown in Table 2.

\section{Cortical thickness analysis}

MDD patients showed a significantly (corrected for multiple comparisons with Bonferroni correction) thinner cortex in the left OFC $\left(F_{(1,71)}=7.941, p=0.006\right.$; Table 3, Fig. 2), and right OFC $\left(F_{(1,71)}=17.447, p<0.001\right.$; Table 3, Fig. 2). For the sub-region analysis, MDD patients

Table 1 Demographic and clinical characteristics of medication-naïve female patients with MDD and HCs

\begin{tabular}{lllll}
\hline Demographic data & MDD patients & HCs & F & $P$ \\
\hline N & 31 & 43 & - & - \\
Age, years & $40.83(9.69)$ & $43.51(12.21)$ & 1.020 & 0.316 \\
$\begin{array}{l}\text { Illness duration, } \\
\quad \text { months }\end{array}$ & $8.51(20.02)$ & - & - & - \\
Education, years & $12.41(3.29)$ & $13.88(3.23)$ & 3.640 & 0.060 \\
HDRS-17 & $20.96(5.09)$ & $2.41(2.20)$ & 453.768 & $<0.001^{*}$ \\
& & & &
\end{tabular}

All data are given as mean (standard deviation)

MDD major depressive disorder, HCs healthy controls, HDRS-17 17-item Hamilton Depression Rating Scale

* Significant $p$ value; $p<0.05$ showed a significantly (corrected for multiple comparisons with Bonferroni correction) thinner cortex in the left medial OFC $\left(F_{(1,71)}=8.117, p=0.006\right.$; Table 3, Fig. 2), right medial OFC $\left(F_{(1,71)}=21.795, p<0.001\right.$; Table 3 , Fig. 2) and right lateral OFC $\left(F_{(1,71)}=9.932, p=0.002\right.$; Table 3, Fig. 2) compared to HCs. No significant interaction of diagnosis (MDD patients, HCs) and MAOAuVNTR genotype (MAOA-H, MAOA-L) on OFC cortical thickness was revealed for any of the regions (left OFC $\left(F_{(1,69)}=0.345, p=0.559\right)$, right $\operatorname{OFC}\left(F_{(1,69)}=0.010\right.$, $p=0.920)$, left medial OFC $\left(F_{(1,69)}=1.544, p=0.218\right)$, right medial OFC $\left(F_{(1,69)}=0.509, p=0.478\right)$, left lateral OFC $\left(F_{(1,69)}=0.020, p=0.889\right)$, right lateral OFC $\left(F_{(1,69)}=0.063, p=0.802\right)$; Table 4$)$. Additionally, the interaction of diagnosis and $M A O A-\mathrm{uVNTR}$ genotype was examined on the whole brain both regionally and vertex by vertex. For the regional analysis, 34 cortical ROIs with reference to the Desikan-Killiany atlas were analyzed using ANCOVA with age as a covariate. No significant interaction was revealed after Bonferroni correction. For the vertex by vertex analysis, no significant interaction was observed after Monte Carlo Null-Z Simulation (1.3 threshold, bidirectional) in both hemispheres.

\section{Discussion}

In accordance with our first hypothesis, MDD patients showed a significant reduction in OFC thickness compared to HCs. Our results are similar to previous studies reporting a decrease in OFC thickness in MDD patients $[6,32]$. The OFC acts as a key structure in emotion processing and is considered to be important in tasks such as reward-guided behavior, mood regulation, and impulse control [33]. Regional specialization of the OFC has been reported by previous cytoarchitectonic [34], connectivity [5], and functional studies [35], and the medial 
Table 2 Hardy-Weinberg equilibrium of allele frequencies and genotype distribution of MAOA-uVNTR

\begin{tabular}{|c|c|c|c|c|c|c|c|c|}
\hline & \multicolumn{4}{|c|}{ Hardy-Weinberg equilibrium of allele frequencies } & \multicolumn{4}{|c|}{ Genotype distribution, $\mathrm{N}(\%)$} \\
\hline & $2 \mathrm{R}$ & $3 R$ & $4 \mathrm{R}$ & $\mathbf{P}$ & $2 R / 3 R$ & $3 R / 3 R$ & $3 R / 4 R$ & $4 R / 4 R$ \\
\hline Total & $2(1.35 \%)$ & $97(65.54 \%)$ & $49(33.11 \%)$ & 0.670 & $2(2.70 \%)$ & $30(40.54 \%)$ & $35(47.29 \%)$ & $7(9.45 \%)$ \\
\hline MDD patients & $2(3.23 \%)$ & $39(62.9 \%)$ & $21(33.87 \%)$ & 0.542 & $2(6.45 \%)$ & $13(41.93 \%)$ & $11(35.48 \%)$ & $5(16.12 \%)$ \\
\hline $\mathrm{HCs}$ & $0(0.00 \%)$ & $58(67.44 \%)$ & $28(32.56 \%)$ & 0.075 & $0(0.00 \%)$ & $17(39.53 \%)$ & $24(55.81 \%)$ & $2(4.65 \%)$ \\
\hline
\end{tabular}

MAOA-uVNTR monoamine oxidase A-upstream variable number of tandem repeats, MDD major depressive disorder, $H C s$ healthy controls

Table 3 Averaged cortical thickness $(\mathrm{mm})$ of the OFC in MDD patients and HCs

\begin{tabular}{lllrc}
\hline & \multicolumn{2}{l}{ Cortical thickness } & F & \multicolumn{1}{l}{$\boldsymbol{P}$} \\
\cline { 2 - 3 } & MDD patients & HCs & & \\
\hline Left OFC & $2.49(0.14)$ & $2.56(0.16)$ & 7.941 & $0.006^{*}$ \\
Right OFC & $2.50(0.14)$ & $2.63(0.17)$ & 17.447 & $<0.001^{*}$ \\
Left medial OFC & $2.43(0.13)$ & $2.53(0.20)$ & 8.117 & $0.006^{* *}$ \\
Left lateral OFC & $2.53(0.16)$ & $2.58(0.17)$ & 3.971 & 0.050 \\
Right medial OFC & $2.38(0.17)$ & $2.57(0.20)$ & 21.795 & $<0.001^{* *}$ \\
Right lateral OFC & $2.57(0.14)$ & $2.68(0.19)$ & 9.932 & $0.002^{* *}$ \\
\hline
\end{tabular}

ANCOVA analysis with age as a covariate. All data are given as mean (standard deviation)

OFC orbitofrontal cortex, MDD major depressive disorder, HCs healthy controls

* Significance level was corrected for multiple comparisons using Bonferroni correction; $p<0.05 / 2=0.025$

** Significance level was corrected for multiple comparisons using Bonferroni correction; $p<0.05 / 4=0.0125$

and lateral parts are assumed to have distinct functions in emotional processing. The lateral OFC is granular [34] and is thought to have evolved from a paleocortical moiety. It is related to medial and dorsal parts of the basal nucleus of the amygdala, as well as to sensory and premotor areas and the posterior cingulate [35]. It is part of the frontostriatal system which is an executive control system, and functionally the lateral OFC has been related to the formation of associations between emotions, especially positive ones, and cognitions [2]. The medial OFC is agranular or dysgranular [34] and is thought to have evolved from an archicortical moiety. It is closely connected to the hippocampal formation, ventrolateral parts of the basal nucleus of the amygdala, dorsolateral prefrontal cortex, and anterior cingulate cortex [2]. Functionally the medial OFC seems to be specialized for emotional processing [35], particularly for negative emotions [34]. Lesions in the OFC have previously been associated with abnormalities in affective behaviors such as depressed mood, anger, affective instability, irritability, and anxiety symptoms [36]. Decreases in cortical thickness, neuronal sizes, and neuronal and glial densities in cortical layers of the OFC in patients with MDD have also been reported [37]. Our results are in accordance with such previous studies reporting structural alterations of the OFC to be a part of the foundation that underlies several clinical features of MDD [2].

Most neuroimaging studies on MDD have been volume based, and relatively fewer studies have investigated the change in cortical thickness of MDD patients compared to HCs [6]. In addition, although various imaging genetics studies have reported changes in cortical thickness of brain areas influenced by certain genetic polymorphisms, most studies were carried out on HCs and not on MDD patients [18]. Our study is meaningful in that we attempted to investigate both the difference in cortical thickness of the OFC between medication-naïve MDD patients and $\mathrm{HCs}$, and the interaction of diagnosis and genotype on OFC thickness. However, despite the fact that previous studies on healthy controls have reported a decrease in lateral OFC thickness [17] and an increase in OFC volume in $M A O A$-L individuals [16], we did not detect a significant association between $M A O A$-uVNTR polymorphism and OFC thickness in MDD.

Considered a limitation, we relied on a relatively small sample size, which may have influenced our negative finding on diagnosis and genotype interaction on OFC thickness. While our sample size was similar to those of recent neuroimaging studies, future studies including a larger sample size may be helpful in demonstrating more confident results. A recent study on sample size estimation of cortical thickness analysis suggested that 50 subjects in each group are required to reliably detect cortical thickness changes of $0.25 \mathrm{~mm}$ over $95 \%$ of the entire cortical surface with surface-based smoothing of 10-mm FWHM [38]. However, there is still no consensus on sample size estimation in analyses derived from cortical modeling procedure performed by FreeSurfer. In addition, although we purposely included only female subjects for reasons previously stated, the fact that all subjects were females may be considered as a limitation in the context of generalizing our findings. The potential neuroprotective effect of estrogen has been suggested by previous studies [39], and $M A O A$ genotype-dependent structural changes in the OFC have previously been observed in males but not females [16, 17]. Moreover, studies on MAOA genotype variation have persistently 


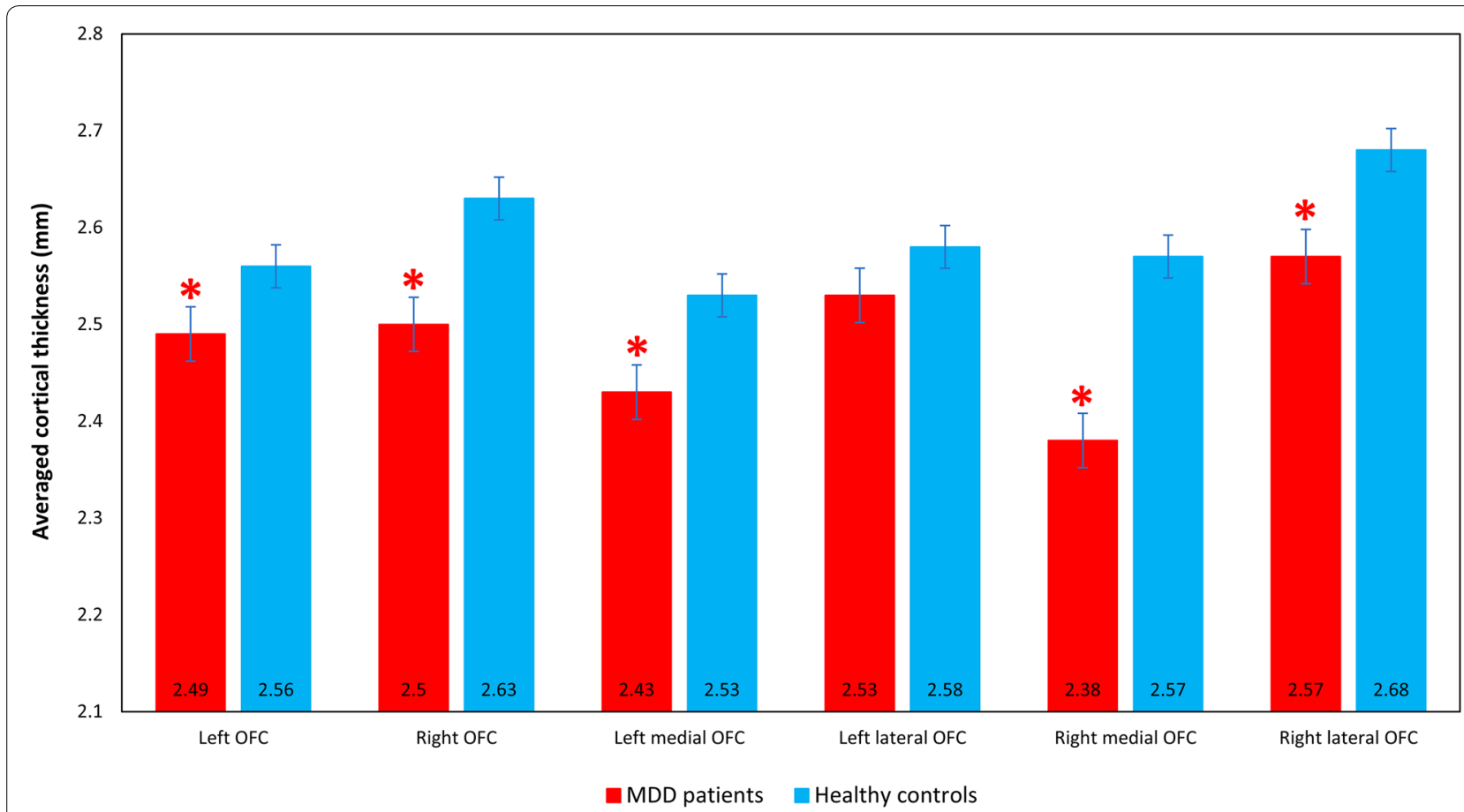

Fig. 2 Averaged cortical thickness of the orbitofrontal cortex (OFC) in major depressive disorder (MDD) patients and healthy controls (HCs), as automatically parcelated by FreeSurfer. The red bars represent the average cortical thickness of MDD patients in each OFC area. The blue bars represent the average cortical thickness of HCs in each OFC area. MDD patients show a significantly thinner cortex in the left OFC, right OFC, left medial OFC, right medial OFC and right lateral OFC compared to HCs

Table 4 Interaction of diagnosis (MDD patients, HCs) and MAOA-uVNTR genotype (MAOA-H, MAOA-L) on OFC cortical thickness

\begin{tabular}{lllrl}
\hline & $\mathbf{F}$ & \multicolumn{1}{l}{$\boldsymbol{P}$} & \multicolumn{1}{l}{$\boldsymbol{\eta}_{\mathbf{p}}^{\mathbf{2}}$} & Observed power \\
\hline Left OFC & 0.345 & 0.559 & 0.005 & 0.089 \\
Right OFC & 0.010 & 0.920 & $<0.001$ & 0.051 \\
Left medial OFC & 1.544 & 0.218 & 0.022 & 0.232 \\
Left lateral OFC & 0.020 & 0.889 & $<0.001$ & 0.052 \\
Right medial OFC & 0.509 & 0.478 & 0.007 & 0.108 \\
Right lateral OFC & 0.063 & 0.802 & 0.001 & 0.057 \\
\hline
\end{tabular}

ANCOVA analysis with age as a covariate

MDD major depressive disorder, HCs healthy controls, MAOA-uVNTR monoamine oxidase A-upstream variable number of tandem repeats, OFC orbitofrontal cortex

reported a greater vulnerability of men to the effects of the $M A O A-\mathrm{L}$ on brain structure, function and connectivity. However, as males with MDD have been reported to have more pronounced neuroanatomic alterations compared to females with MDD, and this in turn could be interpreted as males needing a more pronounced brain structural alteration to develop depressive symptoms [2], our results could be considered even more meaningful as we observed cortical thickness change in subjects composed only of females. Furthermore, whereas males are hemizygous carriers of either one $M A O A$ allele, females are heterozygotes. As MAOAuVNTR polymorphism maps to an $\mathrm{X}$ chromosome region suspected to escape the normal $\mathrm{X}$ chromosome inactivation [40], it is difficult to compare heterozygous females in terms of enzymatic activity. However, similar to our study, previous studies have divided females into two main groups based on their MAOA-uVNTR genotype: $M A O A-\mathrm{L}$ (subjects homozygous for the shortest uVNTR allele) and MAOA-H (subjects homozygous for long uVNTR alleles, together with subjects heterozygous for a long and the shortest allele) [9]. Finally, the use of only one locus of the $M A O A$ gene may be considered a limitation, and a validation study using an independent case-control cohort may be needed to solidify the conclusion drawn from our results. Previous imaging genetics studies have investigated numerous single-nucleotide polymorphisms of a certain gene and their association with brain structure [41]. Future studies which investigate comprehensively the association between various genetic variations of $M A O A$ and structural alterations of the OFC will help shed light on the influence the $M A O A$ gene has on OFC structure. 


\section{Conclusions}

In conclusion, although the OFC is not the only brain region implicated in depression, our results do suggest that structural alterations of the OFC may be associated with the pathophysiology of MDD. Future studies with larger sample sizes are needed to detect a possible association between $M A O A-\mathrm{uVNTR}$ genotype and OFC thickness in depression, which will help investigate the mechanistic hypotheses motivated by our results.

\begin{abstract}
Abbreviations
MAOA-UVNTR: monoamine oxidase A-upstream variable number of tandem repeats; MDD: major depressive disorder; OFC: orbitofrontal cortex; MAOA-H: high-activity monoamine oxidase A-upstream variable number of tandem repeats variant; MAOA-L: low-activity monoamine oxidase A-upstream variable number of tandem repeats variants; HCs: healthy controls; DSM-IV-TR: Diagnostic and Statistical Manual for Mental Disorders-IV-Text Revision; HRDS: Hamilton Depression Rating Scale; MRI: magnetic resonance imaging; ROI: region of interest.
\end{abstract}

\section{Authors' contributions}

EW managed the literature searches and wrote the first draft. EW and SC undertook the statistical analyses of the data. SC and JK contributed to the analyses and interpretations of the MRI data. $\mathrm{BH}$ and $\mathrm{ML}$ designed the study and $\mathrm{BH}$ wrote the protocol as a corresponding author. All authors read and approved the final manuscript.

\section{Author details}

1 Department of Psychiatry, Korea University Anam Hospital, Korea University College of Medicine, 73, Inchon-ro, Seongbuk-gu, Seoul 136-705, Republic of Korea. ${ }^{2}$ Department of Brain and Cognitive Engineering, Korea University, Seoul, Republic of Korea. ${ }^{3}$ Department of Biomedical Science, Korea University, Seoul, Republic of Korea. ${ }^{4}$ Department of Psychiatry, Korea University Anam Hospital, Korea University College of Medicine, Seoul, Republic of Korea.

\section{Acknowledgements}

Not applicable.

\section{Competing interests}

The authors declare that they have no competing interests.

\section{Availability of data and materials}

The datasets during and/or analyzed during the current study are available from the corresponding author on reasonable request.

\section{Consent for publication}

Consent for publication has been obtained from all participants.

\section{Funding}

This work was supported by the Basic Science Research Program through the National Research Foundation of Korea (NRF) funded by the Ministry of Education, Science and Technology (NRF-2011-0023272) and the Korean Health Technology R\&D Project, Ministry of Health and Welfare, Republic of Korea (HI12C0003). The funder had no role in the design of the study and collection, analysis, and interpretation of data and in writing the manuscript.

\section{Ethics approval and consent to participate}

The study protocol was approved by the institutional review board of Korea University College of Medicine and signed informed consent was obtained from all participants according to the Declaration of Helsinki.

Received: 14 July 2016 Accepted: 19 September 2016

Published online: 12 October 2016
References

1. Konarski JZ, McIntyre RS, Kennedy SH, Rafi-Tari S, Soczynska JK, Ketter TA. Volumetric neuroimaging investigations in mood disorders: bipolar disorder versus major depressive disorder. Bipolar Disord. 2008;10:1-37.

2. Lacerda AL, Keshavan MS, Hardan AY, Yorbik O, Brambilla P, Sassi RB, et al. Anatomic evaluation of the orbitofrontal cortex in major depressive disorder. Biol Psychiatr. 2004;55:353-8.

3. Bremner JD, Vythilingam M, Vermetten E, Nazeer A, Adil J, Khan S, et al. Reduced volume of orbitofrontal cortex in major depression. Biol Psychiatr. 2002;51:273-9.

4. Vasic N, Walter H, Hose A, Wolf RC. Gray matter reduction associated with psychopathology and cognitive dysfunction in unipolar depression: a voxel-based morphometry study. J Affect Disord. 2008;109:107-16.

5. Carmichael ST, Price JL. Limbic connections of the orbital and medial prefrontal cortex in macaque monkeys. J Comp Neurol. 1995;363:615-41.

6. Tu PC, Chen LF, Hsieh JC, Bai YM, Li CT, Su TP. Regional cortical thinning in patients with major depressive disorder: a surface-based morphometry study. Psychiatr Res. 2012;202:206-13.

7. Grieve SM, Korgaonkar MS, Koslow SH, Gordon E, Williams LM. Widespread reductions in gray matter volume in depression. Neuroimage Clin. 2013;3:332-9

8. Meyer-Lindenberg A. Intermediate or brainless phenotypes for psychiatric research? Psychol Med. 2010;40:1057-62.

9. Melas PA, Wei Y, Wong CC, Sjoholm LK, Aberg E, Mill J, et al. Genetic and epigenetic associations of MAOA and NR3C 1 with depression and childhood adversities. Int J Neuropsychopharmacol. 2013;16:1513-28.

10. Zhang J, Chen Y, Zhang K, Yang H, Sun Y, Fang Y, et al. A cis-phase interaction study of genetic variants within the MAOA gene in major depressive disorder. Biol Psychiatr. 2010;68:795-800.

11. Williams LM, Gatt JM, Kuan SA, Dobson-Stone C, Palmer DM, Paul RH, et al. A polymorphism of the MAOA gene is associated with emotional brain markers and personality traits on an antisocial index. Neuropsychopharmacology. 2009;34:1797-809.

12. Yu YW, Tsai SJ, Hong CJ, Chen TJ, Chen MC, Yang CW. Association study of a monoamine oxidase a gene promoter polymorphism with major depressive disorder and antidepressant response. Neuropsychopharmacology. 2005;30:1719-23.

13. Huang SY, Lin MT, Lin WW, Huang CC, Shy MJ, Lu RB. Association of monoamine oxidase A (MAOA) polymorphisms and clinical subgroups of major depressive disorders in the Han Chinese population. World J Biol Psychiatr. 2009;10:544-51.

14. Won E, Ham BJ. Imaging genetics studies on monoaminergic genes in major depressive disorder. Prog Neuropsychopharmacol Biol Psychiatr. 2016:64:311-9.

15. Shih JC, Thompson RF. Monoamine oxidase in neuropsychiatry and behavior. Am J Hum Genet. 1999;65:593-8.

16. Meyer-Lindenberg A, Buckholtz JW, Kolachana B, Hariri AR, Pezawas $L$, Blasi G, et al. Neural mechanisms of genetic risk for impulsivity and violence in humans. Proc Natl Acad Sci USA. 2006;103:6269-74.

17. Cerasa A, Gioia MC, Labate A, Lanza P, Magariello A, Muglia M, et al. MAO A VNTR polymorphism and variation in human morphology: a VBM study. Neuroreport. 2008;19:1107-10.

18. Cerasa A, Cherubini A, Quattrone A, Gioia MC, Magariello A, Muglia M, et al. Morphological correlates of MAO A VNTR polymorphism: new evidence from cortical thickness measurement. Behav Brain Res. 2010;211:118-24.

19. Fjell AM, Walhovd KB. Structural brain changes in aging: courses, causes and cognitive consequences. Rev Neurosci. 2010;21:187-221.

20. Scott ML, Bromiley PA, Thacker NA, Hutchinson CE, Jackson A. A fast, model-independent method for cerebral cortical thickness estimation using MRI. Med Image Anal. 2009;13:269-85.

21. Wagner G, Schultz CC, Koch K, Schachtzabel C, Sauer H, Schlosser RG. Prefrontal cortical thickness in depressed patients with high-risk for suicidal behavior. J Psychiatr Res. 2012;46:1449-55.

22. Winkler AM, Kochunov P, Blangero J, Almasy L, Zilles K, Fox PT, et al. Cortical thickness or grey matter volume? The importance of selecting the phenotype for imaging genetics studies. Neuroimage. 2010;53:1135-46.

23. Han X, Jovicich J, Salat D, van der Kouwe A, Quinn B, Czanner S, et al. Reliability of MRI-derived measurements of human cerebral cortical thickness: the effects of field strength, scanner upgrade and manufacturer. Neuroimage. 2006;32:180-94. 
24. Duman RS, Monteggia LM. A neurotrophic model for stress-related mood disorders. Biol Psychiatr. 2006;59:1116-27.

25. Reif A, Weber H, Domschke K, Klauke B, Baumann C, Jacob CP, et al. Meta-analysis argues for a female-specific role of MAOA-uVNTR in panic disorder in four European populations. Am J Med Genet Part B. 2012;159B:786-93.

26. Fischl B, van der Kouwe A, Destrieux C, Halgren E, Segonne F, Salat DH, et al. Automatically parcellating the human cerebral cortex. Cereb Cortex. 2004;14:11-22.

27. Fischl B, Dale AM. Measuring the thickness of the human cerebral cortex from magnetic resonance images. Proc Natl Acad Sci USA. 2000;97:11050-5.

28. Dale AM, Fischl B, Sereno MI. Cortical surface-based analysis. I. Segmentation and surface reconstruction. Neuroimage. 1999;9:179-94.

29. Segonne F, Pacheco J, Fischl B. Geometrically accurate topology-correction of cortical surfaces using nonseparating loops. IEEE Trans Med Imaging. 2007;26:518-29.

30. Desikan RS, Segonne F, Fischl B, Quinn BT, Dickerson BC, Blacker D, et al. An automated labeling system for subdividing the human cerebral cortex on MRI scans into gyral based regions of interest. Neuroimage. 2006;31:968-80

31. Manor I, Tyano S, Mel E, Eisenberg J, Bachner-Melman R, Kotler M, et al. Family-based and association studies of monoamine oxidase A and attention deficit hyperactivity disorder (ADHD): preferential transmission of the long promoter-region repeat and its association with impaired performance on a continuous performance test (TOVA). Mol Psychiatr. 2002;7:626-32.

32. van Eijndhoven $P$, van Wingen $G$, Katzenbauer M, Groen W, Tepest R, Fernandez $\mathrm{G}$, et al. Paralimbic cortical thickness in first-episode depression: evidence for trait-related differences in mood regulation. Am J Psychiatr. 2013;170:1477-86.

33. Price JL. Prefrontal cortical networks related to visceral function and mood. Ann N Y Acad Sci. 1999:877:383-96.

34. Morecraft RJ, Geula C, Mesulam MM. Cytoarchitecture and neural afferents of orbitofrontal cortex in the brain of the monkey. J Comp Neurol. 1992:323:341-58.

35. Northoff G, Richter A, Gessner M, Schlagenhauf F, Fell J, Baumgart F, et al. Functional dissociation between medial and lateral prefrontal cortical spatiotemporal activation in negative and positive emotions: a combined fMRI/MEG study. Cereb Cortex. 2000;10:93-107.

36. Grafman J, Schwab K, Warden D, Pridgen A, Brown HR, Salazar AM. Frontal lobe injuries, violence, and aggression: a report of the Vietnam Head Injury Study. Neurology. 1996;46:1231-8.

37. Rajkowska G, Miguel-Hidalgo JJ, Wei J, Dilley G, Pittman SD, Meltzer $\mathrm{HY}$, et al. Morphometric evidence for neuronal and glial prefrontal cell pathology in major depression. Biol Psychiatr. 1999;45:1085-98.

38. Pardoe HR, Abbott DF, Jackson GD. Sample size estimates for wellpowered cross-sectional cortical thickness studies. Hum Brain Mapp. 2013;34:3000-9.

39. Alkayed NJ, Harukuni I, Kimes AS, London ED, Traystman RJ, Hurn PD. Gender-linked brain injury in experimental stroke. Stroke. 1998;29:159-65

40. Carrel L, Cottle AA, Goglin KC, Willard HF. A first-generation X-inactivation profile of the human X chromosome. Proc Natl Acad Sci USA. 1999;96:14440-4

41. Zhang DF, Li J, Wu H, Cui Y, Bi R, Zhou HJ, et al. CFH variants affect structural and functional brain changes and genetic risk of alzheimer's disease. Neuropsychopharmacology. 2016;41:1034-45.

\section{Submit your next manuscript to BioMed Central and we will help you at every step:}

- We accept pre-submission inquiries

- Our selector tool helps you to find the most relevant journal

- We provide round the clock customer support

- Convenient online submission

- Thorough peer review

- Inclusion in PubMed and all major indexing services

- Maximum visibility for your research

Submit your manuscript at www.biomedcentral.com/submit 